\title{
INFLAMMATORY ACNE IN THE ASIAN SKIN TYPE III TREATED WITH A SQUARE PULSE, TIME RESOLVED SPECTRAL DISTRIBUTION IPL SYSTEM: A PRELIMINARY STUDY
}

\author{
Geun-Soo Lee MD
}

Drs Woo E Hann's Skin E Laser Clinic, Seoul, South Korea

\begin{abstract}
Background and aims: Acne remains a severe problem for both patients and clinicians. Various approaches using photosurgery and phototherapy have been reported with varying degrees of success and robustness of results. An improved intense pulsed light (IPL) system has become available with interesting beam characteristic which might improve IPL treatment of inflammatory acne in the Asian skin, Fitzpatrick type III/IV.

Subjects and Methods: The 18 study subjects comprised 15 females and 3 males with active mild to moderately severe inflammatory acne (mean age $25.3 \pm 7.70 \mathrm{yr}$, range $17-47 \mathrm{yr}$, Burton scale 1-4, all Fitzpatrick type III Asian skin). They were treated once (8 subjects) or twice (10 subjects) with an IPL system offering both square pulse and time resolved spectral distribution technologies (420 $\mathrm{nm}$ cut-off filter, $30 \mathrm{~ms}$ pulse, $8-12 \mathrm{~J} / \mathrm{cm}^{2}, 2-3$ passes). Clinical photography was taken at baseline and at 4 weeks after the final treatment. Percentage of acne clearance was assessed by an independent dermatological panel and graded from zero to 5, 5 being total clearance.

Results: All subjects completed the study. Post-treatment side effects were mild and transient, with virtually no downtime or postinflammatory hyperpigmentation (PIH) experienced by any subject. All subjects had some improvement and no exacerbation was seen in any subject. Clearance was evaluated by the panel as grade 4 in 5 subjects, grade 3 in 8 , grade 2 in 4 and grade 1 in 1 , so that 14 of 18 subjects (78\%) had clearance of at least 60\%. Patient evaluation was in general slightly better than that of the panel.

Conclusions: The special beam characteristics of the IPL system used in the present preliminary study achieved good to very good results in the treatment of acne in the Fitzpatrick type III Asian skin without PIH induction. The results suggested that acne treatment in the Asian skin using this system is both safe and effective, and merits larger population studies to further optimize parameters and standardize top-up treatments.
\end{abstract}

Key words: Acne vulgaris, endogenous PDT, square pulse, time resolved spectral distribution, Fitzpatrick Asian skin type III

\section{Introduction}

Despite many advances in both the pharmacological armamentarium, and rapid developments in photosurgical/phototherapeutic and non-laser systems, acne vulgaris remains a major problem for dermatologists worldwide. In the United states, it has been reported

Addressee for Correspondence:

Geun-Soo Lee MD

Drs Woo \& Hann's Skin \& Laser Clinic

15-3, Kalwol-dong, Yongsan-gu, Seoul, Korea

Tel: +82-2-754-2590

e-mail: francise@paran.com that $100 \%$ of adolescents will have some form of acne with over 40,000,000 affected, and even over the age of 25 the likelihood of getting acne is still as high as $50 \%$, over $25,000,000$ according to some reports. $1-3$ ) Topical and systemic antibiotics, retinoids, and chemical peelings have been used conventionally for acne treatment with variable success rates. ${ }^{4,5)}$ A recent increase in the antibiotic resistance of Propionibacterium acnes ( $P$. acnes) has caused concern, $\left.{ }^{6}\right)$ and alarms are being raised regarding retinoid-

Manuscript received: June 1st, 2012

Accepted for publication: June 9th, 2012 
associated psychological side effects and their potential ability to cause fetal developmental anomalies. ${ }^{7,8)}$ All of these problems started driving the development of new approaches to the treatment of acne worldwide. 9)

The role of light therapy in the treatment of acne has a long history, and seasonal variations in the severity of the disease led to the connection between sunlight and acne lesion control. 10) This especially appealed to the younger patient who might be less compliant with traditional approaches. At first it was believed that the UV portion of the terrestrial solar spectrum was responsible for improved lesion counts, but this was finally discounted due to the very poor penetration of UV energy into the skin in vivo. ${ }^{11)}$ Coupled with this has been the growing awareness of a link between UV therapy for skin disease and skin cancer formation in later years. ${ }^{12)}$ Attention was drawn to the photodynamic effect of short wave visible blue light in the Soret band on the porphyrins endogenous to $P$. acnes, particularly protoporphyrin IX and coproporphyrin III, with the spectral peak occurring around $415 \mathrm{~nm} .{ }^{13,14)}$ The blue light shifted the porphyrins into their triplet state, and they then reacted with triplet oxygen $\left({ }^{3} \mathrm{O} 2\right)$ to produce highly cytotoxic singlet oxygen $\left({ }^{1} \mathrm{O} 2\right)$ which induced cell death through apoptosis selectively within the $P$. acnes. ${ }^{15)}$ This led to the ground-breaking paper by Papageorgiou and colleagues which proved the efficacy of light-only therapy in the treatment of acne vulgaris. ${ }^{16)}$ With the advent of the so-called NASA LED, ${ }^{17)}$ LED phototherapy for acne has been well-reported. ${ }^{18,19)}$

This reawakened interest in intense pulsed light (IPL) sources as a potential method of treating acne with light, since the band of polychromatic light produced by the flashlamp extends from the near infrared (near-IR) around $1300 \mathrm{~nm}$ or longer to visible shortwave blue around $400 \mathrm{~nm}$. By placing a cut-off filter between the flashlamp and the target skin, it was possible to produce semiselective bands of light with the shorter wavelengths cut off by the filter. The addition of a cut-off filter could eliminate the unwanted longer near-IR energy, thus reducing the heat produced in the target tissue. However, even with a cut-off filter at 415 $\mathrm{nm}$, the IPL energy is still delivered over all of the wavelengths between the blue and near-IR. Various authors have suggested that IPL systems offer efficacy comparable to lasers or LED sources, but with a lower financial burden to the clinician. ${ }^{20-22)}$ However, the potential for unwanted side effects with IPL treatment was initially reportedly higher in the darker Asian skin types, including Koreans and Japanese type III-IV, including exacerbation of unseen pigmentation, so- called latent melasma. ${ }^{23)}$ These problems were due to high fluences, the greater amount of endogenous epidermal melanin and the high levels of energy at wavelengths in the IPL waveband which are preferentially absorbed in melanin.

A new generation of IPL systems was then developed which had more favorable beam characteristics offering so-called 'square pulsed' beams and even spectral distribution over the entire width of a pulse. 24) This allowed the use of lower fluences, and improved the inherent safety of IPL systems for the treatment of acne in Asian patients with skin types IIIIV. An even later generation combined the square pulse technology with time-resolved spectral distribution, further enhancing IPL system safety and efficacy. The present study was designed to assess the efficacy and safety of one such new system in the treatment of acne vulgaris in Korean patients with Fitzpatrick skin type III.

\section{Subjects and Methods}

\section{Patients}

Eighteen subjects with Fitzpatrick type III Asian skin participated in the study, comprising 15 females and 3 males with active mild to moderately severe inflammatory acne. Ages ranged from 17 to $47 \mathrm{yr}$ with a mean age of $25.3 \pm 7.70 \mathrm{yr}$. There were 3 patients with Burton grade 4 acne, 7 each at grades 3 and 2, and 1 at grade 1. Patient demographics are summarized in Table 1. After having the purpose of the study explained to them, all subjects gave written informed consent to participate in the study and for the use of their clinical photography.

\section{System}

The system used was the SOLARI ${ }^{\mathrm{TM}}$ (Lutronic Corporation, Ilsan, Korea), an IPL-based system offering both square pulse technology and time-resolved spectral distribution. The cut-on filter was at $750 \mathrm{~nm}$, with the cut-off filter at a nominal $420 \mathrm{~nm}$ and Figure 1 gives the typical spectral distribution of this filter. The handpiece had a contact sapphire cooling tip, with an active area of $12 \times 40 \mathrm{~mm}\left(4.8 \mathrm{~cm}^{2}\right)$.

\section{Treatment parameters}

A test treatment was performed for each subject on the preauricular area to assess for excessive erythema. The parameters were a $30 \mathrm{~ms}$ pulse width and $10 \mathrm{~J} / \mathrm{cm}^{2}$ pulse energy, which is, from the author's experience, an ideal starting point for the average type III Korean 


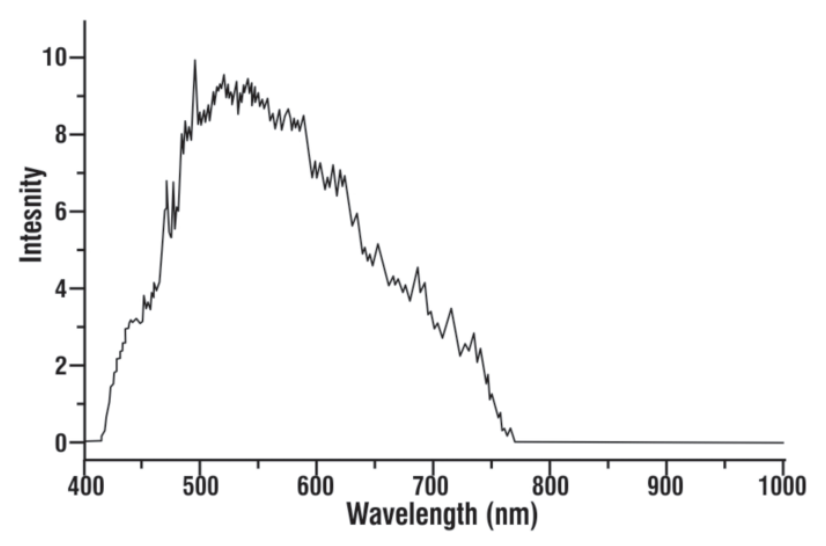

Fig. 1: Spectral distribution of the $420 \mathrm{~nm}$ cut-off, 750 nm cut-on filter supplied with the SOLARI ${ }^{\mathrm{TM}}$ (Lutronic Corporation, Ilsan, South Korea).

skin. In darker skin types, however, such as type III/IV and type IV, the author recommends a maximum of 8 $\mathrm{J} / \mathrm{cm}^{2}$, having experienced two cases of secondary hyperpigmentation when using $10-12 \mathrm{~J} / \mathrm{cm}^{2}$ in these darker skin types.

After setting the desired parameters for each patient based on the test treatment, the cooling tip was placed in contact with the target tissue and IPL energy was delivered over the lesion areas taking time and care to abut the area covered by the sapphire tip to the preceding shot to avoid overlap. In all subjects the 30 $\mathrm{ms}$ pulse width was selected, with a pulse energy of 12 $\mathrm{J} / \mathrm{cm}^{2}$ in $4,10 \mathrm{~J} / \mathrm{cm}^{2}$ in 10 and $8 \mathrm{~J} / \mathrm{cm}^{2}$ in 4 , based on the level of natural pigmentation of the target skin. The cooling tip therefore provided pre- inter- and postpulse cooling. The only area the author avoids treating unless it is absolutely necessary is the forehead, as the thinner layer of tissue over the bone is prone to $\mathrm{PIH}$ formation. When treatment is absolutely required, a lower fluence than the rest of the face should be selected. Three passes were delivered in 16 subjects and 2 passes in the remaining 2. Eight patients had only 1 session, and the remaining 10 had 2 sessions, 2 weeks apart. Individual treatment parameters are seen in Table 1. After each treatment, a cooling mask was applied to maximize patient comfort.

\begin{abstract}
Assessment
Standardized digital photographs were taken at baseline and at 4 weeks after the final treatment. The clinical photography was used by a blinded panel of two independent dermatologists to rate the improvement in the lesion clearance, based on the following 6-grade scale: grade 5, clearance of $85 \%$ and better; 4, 70-84\%; 3, 60-69\%; 2, 40-59\%; 1, 20\%-39\%; and 0, under 20\%. Where there was any difference in the scoring, agreement was reached after discussion. Patients subjectively graded their results using the same 6-grade scale: 5, excellent; 4, very good; 3 , good; 2, fair; 1 , some improvement; and 0 , little or no improvement.
\end{abstract}

Table 1: Patient demographics, treatment parameters and results

\begin{tabular}{|c|c|c|c|c|c|c|c|c|c|c|}
\hline \multirow{2}{*}{$\begin{array}{l}\text { Case } \\
\text { No. }\end{array}$} & \multirow{2}{*}{ Age } & \multirow{2}{*}{ Sex } & \multirow{2}{*}{$\begin{array}{c}\text { Burton } \\
\text { grade }\end{array}$} & \multicolumn{3}{|c|}{ Tx parameters (420 nm filter) } & \multirow{2}{*}{ No of $T x$} & \multirow{2}{*}{$\begin{array}{l}\text { Follow-up } \\
\text { period }\end{array}$} & \multicolumn{2}{|c|}{ Result (Grade) } \\
\hline & & & & Pulse Width (ms) & Fluence $\left(\mathrm{J} / \mathrm{cm}^{2}\right)$ & Passes & & & Panel & Patient \\
\hline 1 & 22 & $\mathrm{~F}$ & 4 & 30 & 10 & 3 & 2 & 2 mnth & 4 & 4 \\
\hline 2 & 24 & $\mathrm{~F}$ & 3 & 30 & 10 & 3 & 1 & 2 mnth & 3 & 4 \\
\hline 3 & 29 & $\mathrm{~F}$ & 3 & 30 & 10 & 3 & 1 & 2 mnth & 2 & 2 \\
\hline 4 & 20 & $\mathrm{~F}$ & 2 & 30 & 12 & 3 & 1 & 3 mnth & 3 & 3 \\
\hline 5 & 29 & $\mathrm{~F}$ & 1 & 30 & 10 & 3 & 2 & 1 mnth & 3 & 3 \\
\hline 6 & 30 & $\mathrm{M}$ & 3 & 30 & 12 & 2 & 2 & 4 mnth & 4 & 4 \\
\hline 7 & 19 & $\mathrm{~F}$ & 4 & 30 & 10 & 3 & 1 & $1 \mathrm{mnth}$ & 4 & 4 \\
\hline 8 & 28 & F & 2 & 30 & 10 & 3 & 2 & 8 mnth & 3 & 3 \\
\hline 9 & 18 & $\mathrm{~F}$ & 4 & 30 & 8 & 3 & 2 & 8 mnth & 2 & 3 \\
\hline 10 & 19 & $\mathrm{~F}$ & 3 & 30 & 8 & 3 & 2 & $8 \mathrm{mnth}$ & 4 & 4 \\
\hline 11 & 20 & $\mathrm{~F}$ & 2 & 30 & 8 & 3 & 2 & $2 \mathrm{mnth}$ & 3 & 4 \\
\hline 12 & 20 & $\mathrm{~F}$ & 2 & 30 & 12 & 3 & 2 & $1 \mathrm{mnth}$ & 4 & 4 \\
\hline 13 & 17 & $\mathrm{M}$ & 3 & 30 & 10 & 3 & 1 & $1 \mathrm{mnth}$ & 3 & 3 \\
\hline 14 & 25 & F & 2 & 30 & 10 & 3 & 1 & $1 \mathrm{mnth}$ & 3 & 3 \\
\hline 15 & 18 & $\mathrm{M}$ & 3 & 30 & 8 & 3 & 1 & $1 \mathrm{mnth}$ & 3 & 4 \\
\hline 16 & 36 & $\mathrm{~F}$ & 3 & 30 & 10 & 3 & 1 & 2 mnth & 4 & 4 \\
\hline 17 & 47 & $\mathrm{~F}$ & 2 & 30 & 10 & 3 & 2 & 2 mnth & 2 & 3 \\
\hline 18 & 34 & $\mathrm{~F}$ & 2 & 30 & 12 & 2 & 2 & 2 mnth & 1 & 2 \\
\hline
\end{tabular}




\section{ORIGINAL ARTICLES}

\section{Results}

All patients completed the study. The results are summarized in Table 1. There were no serious adverse events including secondary hyperpigmentation. Very mild, even erythema was seen in all patients which resolved spontaneously within 24-48 hr, and there was virtually no downtime. The shortest follow-up period was 1 month and the longest 8 months with an average of $2.9 \pm 2.39$ months, and recurrence was minimal, controlled with comedonal scrubs and mild topicals. All patients showed some improvement and exacerbation was not seen in any patient. The panel assessed lesion removal on the 6-grade scale as follows, grade 5 being the highest: No patient scored grade 5 ; grade 4 was seen in 5 subjects, grade 3 in 8 , grade 2 in 4 and grade 1 in 1 , so that 14 of the 18 subjects (78\%) had clearance of at least $60 \%$. The patient scoring was in general slightly higher than the panel's score. No patient graded their result as excellent, grade 4 was scored in 9, grade 3 in 7 and grade 2 in 2 patients. Most patients commented that they felt their overall skin condition had improved, especially those patients in the higher age
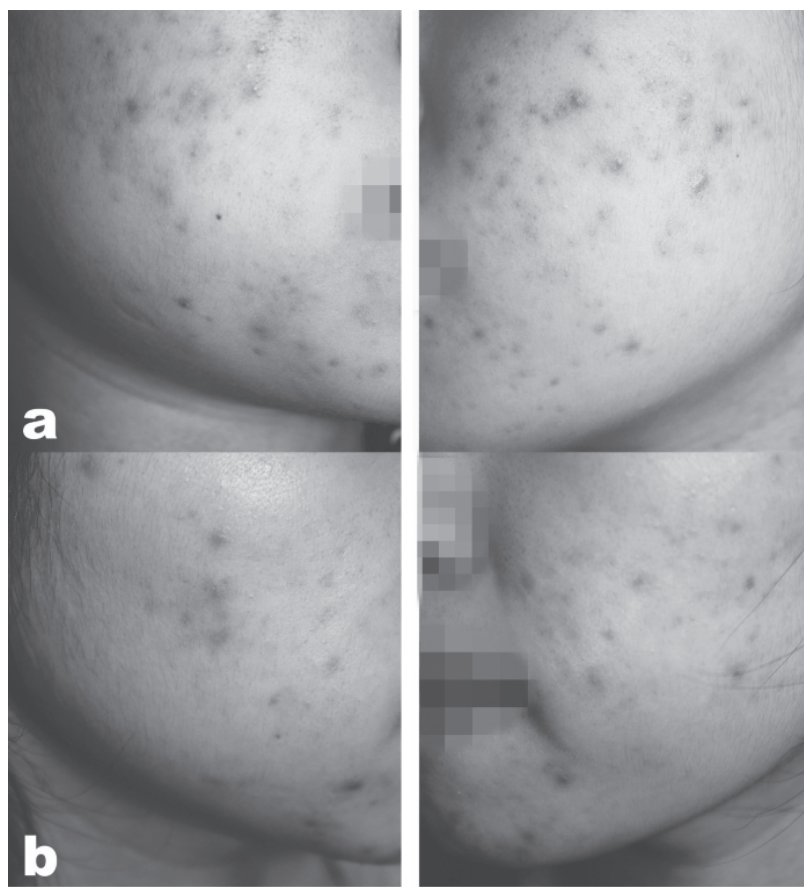

Fig. 2: IPL acne treatment in a representative 19-yearold female patient, Case No 10, (a) at baseline and (b) four weeks after the final treatment. The patient had 2 treatments, 2 weeks between sessions, 3 passes per session, pulse energy of 8 $\mathrm{J} / \mathrm{cm}^{2}$ and a $30 \mathrm{~ms}$ pulsewidth. group. No patient was dissatisfied with the treatment and all patients would be happy to return for repeat treatments in the future. Figures 2 and 3 show representative results in two patients from the study at baseline and at 4 weeks post final treatment.

\section{Discussion}

Even though IPL energy is delivered in a polychromatic burst of intense light, with the addition of suitable precise cut-on and cut-off filters it is possible to produce a band of energy at appropriate wavelengths, especially short wave visible blue in the Soret band which has been shown to inactivate $P$. acnes through endogenous PDT acting on the bacterial porphyrins. 13,19) The waveband produced with the blue cut-off filter in the system used in the present study had a fair portion of its spectral energy in the short wave blue from 410 to $500 \mathrm{~nm}$, the spectrum known to activate porphyrins, and the cut-on filter restricted all wavelengths longer than $750 \mathrm{~nm}$, thus avoiding excess heat being dumped into the tissue from the excluded nearIR component. There was also a postulated benefit
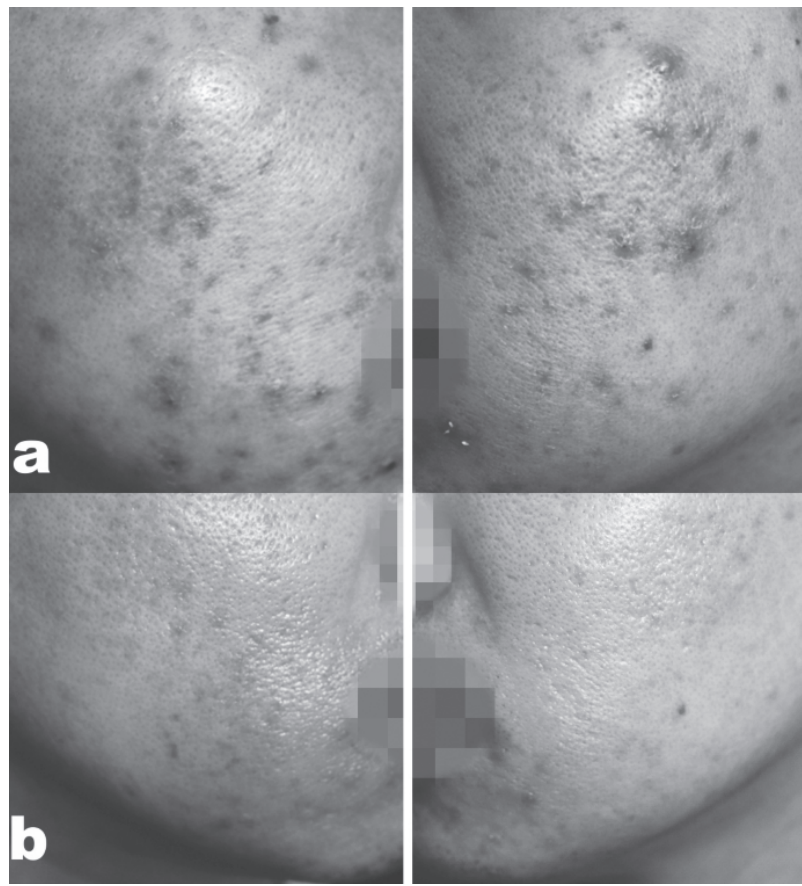

Fig. 3: PL acne treatment in a representative 30-yearold male patient, Case No 6, (a) at baseline and (b) four weeks after the final treatment. The patient had 2 treatments, 2 weeks apart, 2 passes per session, pulse energy of $10 \mathrm{~J} / \mathrm{cm}^{2}$ and a $30 \mathrm{~ms}$ pulsewidth 
from the yellow, red and very near-IR wavebands delivered concomitantly with the blue light regarding skin rejuvenation, ${ }^{25,26)}$ and this may well explain why most of the patients commented subjectively on the overall improvement of their skin condition in addition to the improvement in their acne.

The author believes that the specific characteristics of the system used also helped achieve good results with low fluence and few treatments, 1 session in 8 and 2 sessions in the remaining 10 patients. Further treatments may well have improved the results even further, but that is for another study to ascertain. The special characteristics of the beam delivered by this system also probably went a long way in avoiding any PIH in all of the patients treated. However, relying on these characteristics alone is not enough. To help minimize PIH formation, the author recommends a maximum of $8 \mathrm{~J} / \mathrm{cm}^{2}$ for darker skin types than the average type III of the study subjects, and to avoid treating the forehead in all Asian skin types unless it is absolutely required, in which case the pulse energy must be set to a lower level than the rest of the face.

As for the specific characteristics, the system used in the study offered a combination of two interesting technologies: square pulse technology, and timeresolved spectral distribution. Square pulse technology involves keeping the flashlamp capacitors in a simmer mode, known as the constant current approach, rather than allowing them to discharge totally as is the case in the older free discharge technology, so that, when activated, the flashlamp delivers a higher and more even pulse of energy much faster, and without the phenomenon known as "spectral jitter" 27 ) whereby a spectral shift in the lamp output is seen over the duration of the pulse from the infrared to the blue and then back to the red zones of the spectrum, following the spectrogram of light energy emitted by xenon gas in the flashlamp. However, there is a problem during this process: the energy is not evenly distributed, but tends to accumulate unevenly across the emitted waveband. Therefore, filtering is generally adapted to solve this problem to remove the unwanted spectral components so that a high fluence is required to obtain effective treatment, but there is a high risk of photothermal-related complications. In contrast, the square pulse technology has the benefit of even energy distribution within the given pulse duration. In this case, the capacity is almost double that of the free discharge technology. Figure 4 shows the spectral distribution compared between a free discharge system and the current square pulsebased system illustrating the much higher and more even intensity for the same pumping power for the flashlamp. The clinical importance of this is the uneven distribution of power across the beam profile of the conventional type, possibly leading to hot-spot creation and more chance of inducing adverse side effects.

In brief, an IPL system using square pulse technology is able to reduce the side effects of treatment even at high fluences but devices based on the free discharge system need to be used with caution in treatment because of the risk of burns associated with high fluences. Currently, the number of IPL systems with a square pulse is somewhat limited, and additionally the cut-off-filter technique is sometimes not generally well matched with the true spectral output in those systems. In time-resolved spectral distribution, ${ }^{24)}$ each pulse in a conventional system consists of a series of different spectral energy components, rather like mismatched
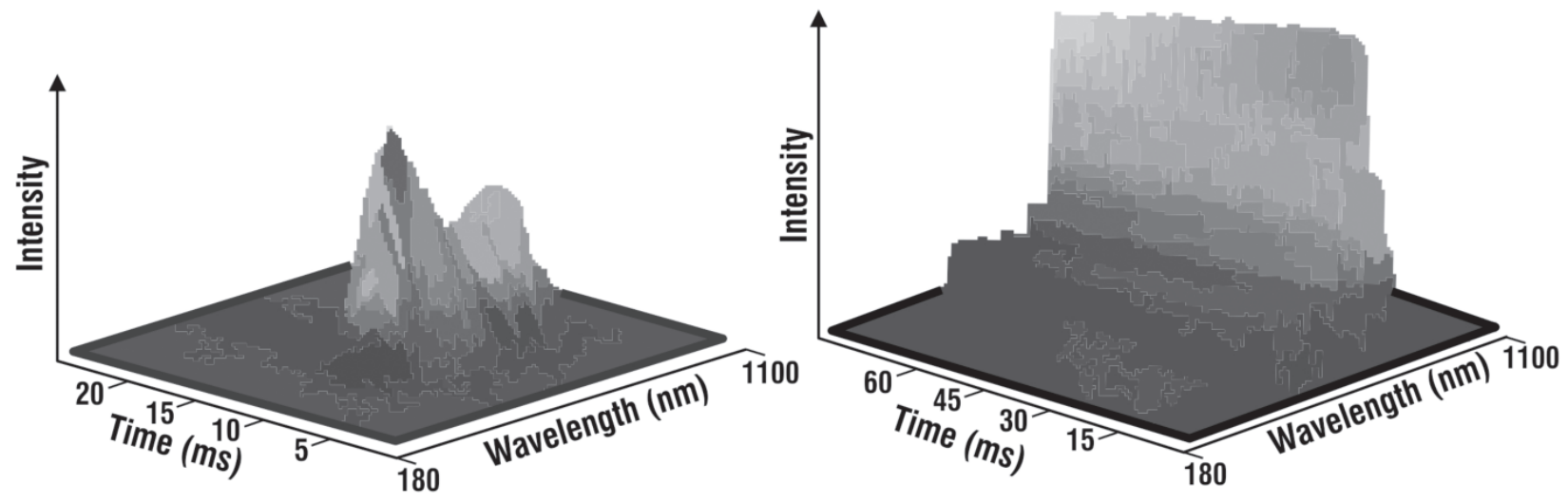

Fig. 4: Representative illustrations of IPL shots from a free discharge system (left) and a square pulse, constant current system (right). The power distribution across the pulse is extremely uneven and short in the free discharge example, whereas the square pulse mode offers even discharge across all wavelengths. 
micropulses making up the macropulse even though it is a true single pulse or subpulse. In the case of time resolution of the spectral distribution, the energy is evenly distributed across the spectrum for each subpulse, so they are identical from start to finish. Once again, this aids homogeneity of the energy across the beam, leading to dependable treatment results. Figure $\mathbf{5}$ illustrates this difference with spectral distribution measurement versus intensity, and also shows the result of the effective cut-on and cut-off filters employed in the present system. The combination of square pulse and time-resolved spectral distribution allows the clinician to keep an appropriate distance between subpulses, to deliver even photothermal energy, to maintain the same pulse duration, and to allow a faster repetition rate between macropulses.

In addition to the above factors, efficient skin cooling also plays an important role in guaranteeing a good result with minimizing unwanted side effects during IPL treatment. The incorporation of an efficient skin cooling system in the handpiece has the benefit of maintaining safe levels of heat in the epidermis thus preventing epidermal damage. An IPL system fitted with effective skin cooling, such as is employed in the system used in the present study, has the advantage of significantly reducing complications as the epidermal surface temperature is well-controlled and remains within the ideal therapeutic range.

\section{Conclusions}

In this preliminary trial, inflammatory acne was welltreated with an IPL system combing the new technologies of square pulse mode and time-resolved spectral distribution, and the good results were further assisted by the precision of the cut-on/cut-off filter and efficient contact cooling crystal incorporated in the handpiece. Although no patient managed a top result grade of 5 from the independent assessment panel, all showed some effect and none had any side effects or downtime. Subjectively, patients were reasonably satisfied with the result, and expressed willingness to return for further treatments. Patient compliance is an important consideration in minimally invasive treatments requiring multiple visits. The good results in this trial warrant further trials to fine tune parameters and assess the result of more treatment sessions on the robustness of this IPL system in the treatment of inflammatory acne, to offer another tool for the dermatologist in their battle against this problematic condition.
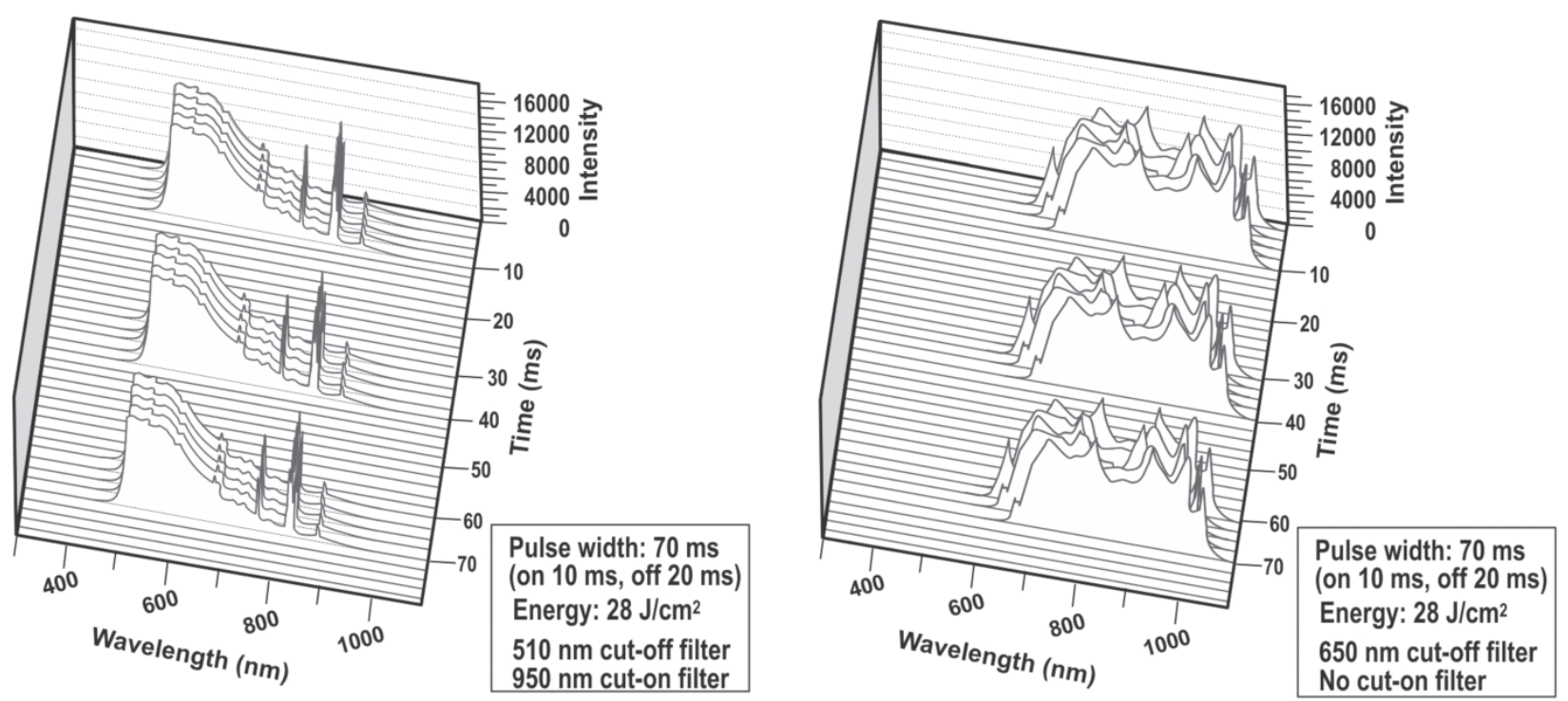

Fig. 5: Printout of representative subpulses from the system used in the present study (left) with time-resolved spectral distribution and a typical system without this technology (right). For both systems the macropulse width was $70 \mathrm{~ms}$, made up of $320 \mathrm{~ms}$ micropulses (subpulses) separated by a $20 \mathrm{~ms}$ interpulse interval, and the pulse energy was $28 \mathrm{~J} / \mathrm{cm}^{2}$. Different cut-off filters were used, $510 \mathrm{~nm}$ for the study system and $650 \mathrm{~nm}$ for the other system. The trial system had an integral cut-on filter at $950 \mathrm{~nm}$, with no cut-on filter for the other system. The absolute regularity of the spectral energy distribution in the subpulses is apparent in the trial system, also giving a very precise cut-off value. On the contrary, the energy distribution across the spectrum in the subpulses for the other system is irregular, potentially leading to unwanted hotspots in the treatment beam with possible side effects such as the 'tiger skin' effect. 


\section{References}

1: Burton JL, Cunliffe WJ, Stafford I, Shuster S (1971): The prevalence of acne vulgaris in adolescence. $\mathrm{Br}$ J Dermatol, 85: 119-126.

2: Leyden JJ (1997): Therapy for acne vulgaris. N Engl J Med, 336: 1156-1162.

3: Mulder MMS, Sigurdsson V, van Zuuren EJ, Klaassen EJ, Faber JAJ, et al.(2001): Psychosocial impact of acne vulgaris. Evaluation of the relation between a change in clinical acne severity and psychosocial state. Dermatology, 203: 124-130.

4: Gollnick HP, Krautheim A (2003): Topical treatment in acne: current status and future aspects. Dermatology, 206: 29-36.

5: Longshore SJ, Hollandsworth K (2003): Acne vulgaris: one treatment does not fit all. Cleve Clin J Med, 70 :672-674.

6: Eady EA, Jones CE, Tipper JL, et al. (1993): Antibiotic resistant propionibacteria in acne: need for policies to modify antibiotic usage. BMJ, 306: 555-556.

7: Stern RS (1989): When a uniquely effective drug is teratogenic: the case of isotretinoin. New Engl J Med, 320: 1007-1009.

8: Hull PR, D'Arcy C (2003): Isotretinoin use and subsequent depression and suicide: presenting the evidence. Am J Clin Dermatol, 4: 493-505.

9: Kaminsky A (2003): Less common methods to treat acne. Dermatology, 206: 68-73.

10: Gfesser M, Worret WI (1996): Seasonal variations in the severity of acne vulgaris. Int J Dermatol, 35:116-117.

11: Charakida A, Seaton ED, Charakida M, Mouser P, et al. (2004): Phototherapy in the treatment of acne vulgaris: what is its role? Am J Clin Dermatol, 5: 211-216.

12: Archier E, Devaux S, Castela E, Gallini A, et al. (2012): Carcinogenic risks of psoralen UV-A therapy and narrowband UV-B therapy in chronic plaque psoriasis: a systematic literature review. J Eur Acad Dermatol Venereol, 26: Suppl 3 22-31.

13: Arakane K, Ryu A, Hayashi C, Masunaga T, et al. (1996): Singlet oxygen (1 delta g) generation from coproporphyrin in Propionibacterium acnes on irradiation. Biochem Biophys Res Commun, 223: 578-582.

14: Ashkenazi H, Malik Z, Harth Y, Nitzan Y (2003): Eradication of Propionibacterium acnes by its endogenic porphyrins after illumination with high intensity blue light. FEMS Immunol Med Microbiol, 35:17-24.
16: Papageorgiou P, Katsambas A, Chu A (2000): Phototherapy with blue (415 nm) and red (660 $\mathrm{nm})$ light in the treatment of acne vulgaris. $\mathrm{Br} \mathrm{J}$ Dermatol;142: 973-978.

17: Whelan HT, Smits RL Jr., Buchman EV, Whelan NT, Turner SG, Margolis DA, et al. Effect of NASA light-emitting diode irradiation on wound healing. J Clin Laser Med Surg 2001;19:305-314.

18: Goldberg DG and Russell B (2004): Combination blue ( $415 \mathrm{~nm})$ and red (633 nm) LED phototherapy in the treatment of mild to severe acne vulgaris. J Cos Laser Therapy,. 8: 71-75.

19: Lee SY, You CE, and Park MY (2007): Blue and red light combination LED phototherapy for acne vulgaris in patients with skin phototype IV. Lasers Surg Med, 39: 180-188.

20: Ceilley RI (2004): Advances in the topical treatment of acne and rosacea. J Drugs Dermatol, 3: (5 Suppl): S12-22

21: Soltes B (2010): Intense pulsed light therapy. Obstet Gynecol Clin North Am. 37: 489-499

22: Babilas P, Schreml S, Szeimies RM, Landthaler M (2010):Intense pulsed light (IPL): a review: Lasers Surg Med, 42: 93-104

23: Negishi K, Kushikata N, Takeuchi K, Tezuka Y, Wakamatsu S (2006): Photorejuvenation by intense pulsed light with objective measurement of skin color in Japanese patients. Dermatol Surg. 32: 1380-1387.

24: Ash C, Town G, Bjerring P (2008): Lasers Surg Med, 40: 83-92. Relevance of the structure of timeresolved spectral output to light-tissue interaction using intense pulsed light (IPL).

25: Weiss RA, Weiss MA, Geronemus RG, McDaniel DH (2004): A novel non-thermal non-ablative full panel LED photomodulation device for reversal of photoaging: digital microscopic and clinical results in various skin types. J Drugs Dermatol, 3: 605-610.

26: Lee SY, Park KH, Choi JW, Kwon JK, et al. (2007): A prospective, randomized, placebo-controlled, double-blinded, and split-face clinical study on LED phototherapy for skin rejuvenation: Clinical, profilometric, histologic, ultrastructural, and biochemical evaluations and comparison of three different treatment settings. J Photochem Photobiol (B), 88: 51-67

27: Ash C, Town G, Clement M ():Confirmation of spectral jitter: a measured shift in the spectral distribution of intense pulsed light systems using a time-resolved spectrometer during exposure and increased fluence. J Med Eng Technol, 34: 97-107. 